DOI: $10.21105 /$ joss. 01822

\section{Software}

- Review 七

- Repository ca

- Archive [a

Editor: Kevin M. Moerman ¿

\section{Reviewers:}

- @thomakra

- QAtif-Anwer

Submitted: 22 August 2019 Published: 14 January 2020

\section{License}

Authors of papers retain copyright and release the work under a Creative Commons Attribution 4.0 International License (CC-BY).

\title{
SIHR: a MATLAB/GNU Octave toolbox for single image highlight removal
}

\author{
Vítor S. Ramos ${ }^{1}$ \\ 1 Federal University of Rio Grande do Norte, Natal, Brazil
}

\section{Summary}

Single image highlight removal (SIHR) refers to an open problem in computer vision concerning the separation of diffuse and specular reflection components from a single image (Tan, 2014). Briefly, the diffuse component refers to a generally low-parametric reflection that takes the color of the body, while the specular component refers to a higher-frequency reflection that takes the color of the illuminant. Under the dichromatic reflection model (Shafer, 1985), they are linearly additive. It is an intrinsic image decomposition, so it has several applications. Recently, there was an interest renewal in this problem for the objective of image enhancement in visual display systems such as TVs (Yamamoto, Kitajima, \& Kawauchi, 2017).

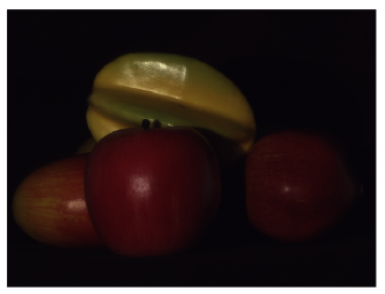

(a)

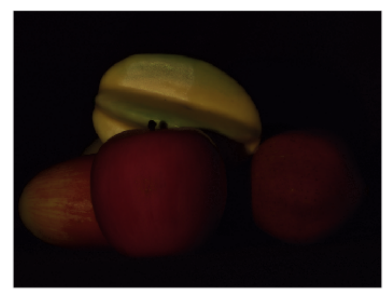

(b)

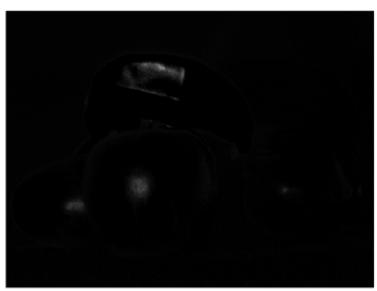

(c)

Figure 1: Example decomposition. (a) Input, (b) diffuse, and (c) specular reflection components

The primary objective of this toolbox is to serve as an aid for ongoing research and development of SIHR methods. Being written in such a high-level language that is MATLAB/GNU Octave allows an easier understanding of the inner workings of these methods. To the best of our knowledge, the resources available to further the understanding of this specific problem are relatively scarce.

Hence, we have started SIHR to implement and gather several different methods from technical literature-starting with the most computationally efficient ones, since the abovementioned systems operate on a limited computing budget and need timely processing. Other methods of interest can be found in recent surveys (Artusi, Banterle, \& Chetverikov, 2011; Khan, Thomas, \& Hardeberg, 2017).

Usage is rather straightforward as the focus of these methods is to work with only a single linear RGB image, i.e. an $m \times n \times 3$ matrix. For uniformity, we ask the image to be doublevalued. In SIHR, calls are simply $I_{-} d=\operatorname{AuthorYEAR}(I)$; , in which $I$ is the original image and $I_{-} d$ is the diffuse component estimate calculated by the AuthorYEAR method. The specular component is $I_{-} s=I-I_{-} d$;

At the time of writing, the methods listed in Table 1 are available. We refer to the SIHR documentation for the latest list of methods available. 
Table 1: List of methods in SIHR

\begin{tabular}{ccccc}
\hline Function & Method & PSNR (dB) & SSIM & Runtime (s) \\
\hline Tan2005 & Tan \& Ikeuchi (2005) & 29.5 & 0.888 & 160 \\
Yoon2006 & Yoon, Choi, \& Kweon (2006) & 34.2 & 0.964 & 2.6 \\
Shen2008 & Shen, Zhang, Shao, \& Xin (2008) & 35.5 & 0.960 & 4.7 \\
Shen2009 & Shen \& Cai (2009) & 35.8 & 0.970 & 0.26 \\
Yang2010 & Yang, Wang, \& Ahuja (2010) & 35.9 & 0.925 & 0.16 \\
Shen2013 & Shen \& Zheng (2013) & 36.7 & 0.960 & 0.063 \\
Akashi2016 & Akashi \& Okatani (2016) & 34.3 & 0.856 & 180 \\
\hline
\end{tabular}

Table 2: List of improvements in SIHR

\begin{tabular}{cc}
\hline Function & Method \\
\hline Yamamoto2019 & Yamamoto \& Nakazawa (2019) \\
\hline
\end{tabular}

Figure 1 presents an actual result of a method from technical literature which was implemented in SIHR. In Table 1, the reproduced metrics are from the Shen \& Zheng (2013) dataset, and were averaged-for each metric-for all four images.

SIHR aims to be a continuous project and welcomes community contributions.

\section{References}

Akashi, Y., \& Okatani, T. (2016). Separation of reflection components by sparse non-negative matrix factorization. Computer Vision and Image Understanding, 146, 77-85. doi:10. 1016/j.cviu.2015.09.001

Artusi, A., Banterle, F., \& Chetverikov, D. (2011). A survey of specularity removal methods. Computer Graphics Forum, 30(8), 2208-2230. doi:10.1111/j.1467-8659.2011.01971.x

Khan, H. A., Thomas, J.-B., \& Hardeberg, J. Y. (2017). Analytical survey of highlight detection in color and spectral images. In Lecture notes in computer science (pp. 197208). Springer International Publishing. doi:10.1007/978-3-319-56010-6_17

Shafer, S. A. (1985). Using color to separate reflection components. Color Research \& Application, 10(4), 210-218. doi:10.1002/col.5080100409

Shen, H.-L., \& Cai, Q.-Y. (2009). Simple and efficient method for specularity removal in an image. Applied Optics, 48(14), 2711. doi:10.1364/ao.48.002711

Shen, H.-L., Zhang, H.-G., Shao, S.-J., \& Xin, J. H. (2008). Chromaticity-based separation of reflection components in a single image. Pattern Recognition, 41(8), 2461-2469. doi:10. 1016/j.patcog.2008.01.026

Shen, H.-L., \& Zheng, Z.-H. (2013). Real-time highlight removal using intensity ratio. Applied Optics, 52(19), 4483. doi:10.1364/ao.52.004483

Tan, R. T. (2014). Specularity, specular reflectance. In Computer vision (pp. 750-752). Springer US. doi:10.1007/978-0-387-31439-6_538

Tan, R. T., \& Ikeuchi, K. (2005). Separating reflection components of textured surfaces using a single image. IEEE Transactions on Pattern Analysis and Machine Intelligence, 27(2), 178-193. doi:10.1109/tpami.2005.36 
Yamamoto, T., Kitajima, T., \& Kawauchi, R. (2017). Efficient improvement method for separation of reflection components based on an energy function. In 2017 IEEE international conference on image processing (ICIP). IEEE. doi:10.1109/icip.2017.8297078

Yamamoto, T., \& Nakazawa, A. (2019). General improvement method of specular component separation using high-emphasis filter and similarity function. ITE Transactions on Media Technology and Applications, 7(2), 92-102. doi:10.3169/mta.7.92

Yang, Q., Wang, S., \& Ahuja, N. (2010). Real-time specular highlight removal using bilateral filtering. In Computer vision ECCV 2010 (pp. 87-100). Springer Berlin Heidelberg. doi:10.1007/978-3-642-15561-1_7

Yoon, K.-j., Choi, Y., \& Kweon, I. S. (2006). Fast separation of reflection components using a specularity-invariant image representation. In 2006 international conference on image processing. IEEE. doi:10.1109/icip.2006.312650 